\title{
THE BUILT ENVIRONMENT'S INFLUENCE ON RESILIENCE OF HEALTHCARE SERVICES: LESSONS LEARNT FROM THE COVID-19 PANDEMIC
}

\author{
Natália Ransolin', Carlos Emilio Stigler Marczyk², Rafael Parmeggiani Gering 3 , \\ Tarcísio Abreu Saurin ${ }^{4}$, Carlos Torres Formoso ${ }^{5}$, and Tor Olav Grøtan ${ }^{6}$
}

\begin{abstract}
The COVID-19 pandemic has posed unprecedented challenges for healthcare services, which have been forced to upscale their capacity to cope with successive surges in demand. The adjustments to match capacity to demand and deal with a new disease have involved creativity and solutions that were not part of the pre-pandemic standardized operating procedures. Those changes are considered manifestations of resilience. This paper focuses on the role played by the built environment of healthcare services during the pandemic, in terms of how it is integral to resilient performance. As such, we investigated the experience of a leading private hospital in Brazil, documenting the main changes related to the built environment and how they influenced resilience. Data collection involved eight interviews with hospital staff. A content analysis allowed the development of a generic functional model of the patient journey and the identification of ten resilience practices. Based on this, six lessons learnt were devised. These lessons are expected to be useful for the design and use of the built environment, supporting the resilience of services.
\end{abstract}

\section{KEYWORDS}

Healthcare services, complexity, resilience, COVID-19, built environment.

1 PhD Candidate, Civil Engineering Post-Graduate Program: Construction and Infrastructure, Federal University of Rio Grande do Sul (UFRGS), Porto Alegre, Brazil, natalia.ransolin@ufrgs.br, orcid.org/0000-0002-7128-8000

2 Master Student, Civil Engineering Post-Graduate Program: Construction and Infrastructure, Federal University of Rio Grande do Sul (UFRGS), Porto Alegre, Brazil, carlosmarczyk@gmail.com, orcid.org/0000-0003-0822-6787

3 Master Student, Civil Engineering Post-Graduate Program: Construction and Infrastructure, Federal University of Rio Grande do Sul (UFRGS), Porto Alegre, Brazil, rafael.gering@ufrgs.br, orcid.org/0000-0003-1078-8906

4 Professor, Industrial Engineering Post-Graduate Program, Federal University of Rio Grande do Sul (UFRGS), Porto Alegre, Brazil, saurin@ ufrgs.br, orcid.org/0000-0003-2929-5888

5 Professor, Civil Engineering Post-Graduate Program: Construction and Infrastructure, Federal University of Rio Grande do Sul (UFRGS), Porto Alegre, Brazil, formoso@ ufrgs.br, orcid.org/0000$\underline{0002-4772-3746}$

$6 \quad$ Associate Professor, Norwegian University of Science and Technology (NTNU), Trondheim, Norway, tor.o.grotan@sintef.no, orcid.org/0000-0002-6863-7038 
The built environment's influence on resilience of healthcare services: lessons learnt from the COVID-19 pandemic

\section{INTRODUCTION}

The COVID-19 pandemic has posed unprecedented challenges for healthcare services, such as coping with a very high and volatile demand in face of scarce human and material resources. The built environment plays a key role in this context as a much larger number of patients, staff, and supplies needs to be accommodated in the existing facilities, although expansions and construction of temporary facilities have also been common. A few studies have discussed the role of the built environment of healthcare facilities during the pandemic, although not based on primary data (Capolongo et al., 2020; Keenan, 2020). Furthermore, little empirical evidence has been gathered and analysed based on explicit theoretical frameworks.

This paper uses the lens of resilience engineering (RE), which is concerned with the development of "theories, methods, and tools to deliberately manage the adaptive ability of organizations in order to function effectively and safely" (Hollnagel, 2017). In light of $\mathrm{RE}$, resilient healthcare is the "ability of the healthcare system to adjust its functioning prior to, during, or following changes and disturbances, so that it can sustain required performance under both expected and unexpected conditions" (Hollnagel et al., 2013, p. $\mathrm{XXv}) . \mathrm{RE}$ is a useful perspective as the pandemic has forced healthcare services to build adaptive capacity on the fly, which includes adapting the built environment. The literature linking resilient healthcare and the built environment is scarce, mostly focused on how healthcare facilities and services cope with demand from acute natural disasters such as floods, earthquakes, and short-lived demand spikes (Bosher et al., 2007; Achour and Price, 2010).In addition to these studies, Ransolin et al. (2020b) investigated the implications of the built environment for the resilience of healthcare services during everyday work, in the context of intensive care units (ICUs).

Thus, there is a need for studies on resilience and built environment in the context of chronic and prolonged disasters such as the COVID-19 pandemic. The far reaching impacts of the pandemic across several hospital units and infrastructures (e.g., wards, emergency services, intensive care units, utilities, etc.) have made it clear that the scope of earlier studies on the built environment and resilient healthcare was limited. This gap is explored in this paper through a case study of how a leading private hospital in Brazil has adapted its facilities to cope with the pandemic through the viewpoint of key informants. Lessons learnt from this case study are expected to be useful to other hospitals facing a similar challenge as well as for the design of future facilities. This investigation is relevant to the lean construction community at least for two reasons: (i)design management, which is a traditional lean construction topic, can benefit from the lessons learnt from the pandemic and put more emphasis on the development of theories and practices for the design of more resilient healthcare services; and (ii) the collapse of healthcare services, which has occurred in many places, implies wastes to society at large, including the construction industry - e.g., absenteeism, closures of construction sites.

\section{HEALTHCARE SERVICES AS COMPLEX SOCIO- TECHNICAL SYSTEMS}

Complex Socio-technical Systems (CSSs) have properties such as uncertainty, technical, social and organizational diversity, as well as a large number of elements in dynamic interactions (Cilliers, 1998). These elements involve several stakeholders, technologies, and regulations working collaboratively towards common goals (Hollnagel et al., 2013). Regarding the built environment, healthcare facilities encompass technical aspects such 
as layout of workspaces, equipment, furniture, and utilities that are constantly changing as a result of their interactions with the environment (Ransolin et al., 2020a).

Due to complexity, there is a gap between what people actually do (Work-as-Done WAD) and what they should do according to policies and standard operating procedures (Work-as-Imagined - WAI) (Hollnagel, 2012). The gap between WAI and WAD is also a relevant analytical approach to the built environment, which in practice differs from what is prescribed in regulations and building design. Ransolin et al., (2020a) refer to this as the gap between Built environment-as-Done (BEAD) and Built environment-asImagined (BEAI).

BEAD stems from the resilient performance (e.g., changes in layout, furniture, etc.) of the users of the built environment, either in order to fill out gaps in design or to cope with the variability of everyday work (Ransolin et al., 2020a). Resilient performance is characterized by four interrelated abilities, namely Respond (know what to do), Monitor (know what to look for), Learn (from positive and negative events), and Anticipate (know what to expect) (Hollnagel, 2017). The Functional Resonance Analysis Method (FRAM) is an effective approach to model the interactions between the functions that make up healthcare services, whether or not these functions are directly associated with the four resilience abilities (Clay-Williams et al., 2015). In FRAM, a function corresponds to the activities required to produce a certain outcome. FRAM also allows for the identification of variabilities in individual functions and the understanding of how they propagate across the whole system, producing non-linear effects (Hollnagel, 2012). For these reasons, FRAM is aligned with the nature of healthcare services and has been used for studying that context (Clay-Williams et al., 2015; Ransolin et al., 2020a/b).

\section{RESEARCH METHOD}

\section{DESCRIPTION OF THE STUdied HEALTHCARE FACILITIES}

The hospital investigated is located in Southern Brazil and was chosen based on convenience, as one of the authors works as the infrastructure manager. It is a private institution known as a reference centre for high-complexity and critical cases, which took a leading role in coping with COVID-19 (Polanczyk et al, 2020). The hospital counts on nearly 3,390 physicians and 2,980 allied health professionals. There are also about 1,020 administrative employees. The main building dates from 1921 and has been expanded and renewed multiple times. Nowadays, the facilities spread over $97,912 \mathrm{~m}^{2}$ of built area. There are adult and paediatric emergency departments, two surgical centres, a maternity unit, five adult ICUs ( 55 beds), a paediatric ICU ( 10 beds), a neonatal ICU ( 28 beds), and 15 patient wards (379 inpatient beds).

\section{DATa Collection AND ANALYSIS}

Case study (Yin, 2014) was the research strategy adopted. This choice was due to the exploratory nature of this study, which was interested in investigating new phenomena in a real-world context. Data collection was based on eight semi-structured interviews and a walkthrough ( 1 hospital visit) in the main patient flows. The study was approved by the hospital's ethics committee. Interviews occurred in December 2020, a period in-between pandemic waves of COVID infections in Southern Brazil. The interviewees were: (i) six nurses, of which two had managerial positions and the other four were at the front-line of patient care; (ii) one doctor with a managerial position; and (ii) one infrastructure manager. The interview script encompassed questions related to the functions performed 
The built environment's influence on resilience of healthcare services: lessons learnt from the COVID-19 pandemic

by the interviewee and the changes in the built environment and services as a result of the pandemic. Each interview lasted on average 1 hour and they were audio-recorded and then fully transcribed.

Content analysis (White \& Marsh, 2006) was used for data extraction from interviews. There were two main data analysis themes defined upfront. The first theme referred to the necessary information for the development of a FRAM model (i.e., functions and their description) that encompassed both the care of COVID and non-COVID patients. Daily activities described by the interviewees were interpreted as their WAD and considered as FRAM functions, which are described according to six aspects as follows: input (I), output (O), resources (R), preconditions (P), control (C), and time (T) (Hollnagel, 2012). Functions are coupled to each other when the output of an upstream function provides one or more of the other aspects to a downstream function. A general FRAM function referred to as <Cope with the pandemic $>$ was created in order to encompass the decisions undertaken by the hospital management in charge of the major decisions related to the pandemic. This function encapsulates the organization resilient performance during the pandemic, and its outputs were linked to the other functions through their precondition aspects. According to Hollnagel (2012), a precondition in FRAM corresponds to conditions that must be ready for a function to start.

The second theme referred to the resilience practices to cope with the pandemic. These practices were modelled as the output of <Cope with the pandemic $>$. Two of the authors independently read the transcripts of the interviews and coded them according to the two aforementioned themes. Then, they met to compare their codifications and reached an agreement on the findings. A third author also thoroughly reviewed these codifications and some additional adjustments were made.

The lessons learnt from the pandemic from a built environment perspective were devised based on the FRAM model. These lessons were mostly implicit in the resilience practices that were outputs of <Cope with the pandemic $>$ and they were also related to the four abilities of resilient systems. The lessons learnt were stated in a manner that they could be of interest to other healthcare organizations and not only to the specific studied hospital.

\section{RESULTS}

\section{FRAM MODEL}

The FRAM model is presented in Figure 1. It has 23 functions and encompasses the flow of both COVID and non-COVID patients.

The couplings between the functions, except for <cope with the pandemic>, are not shown in order not to clutter the visual representation of the model. The outputs of this function are described in the next section.

Twelve functions are applicable to both types of patients, even though they are carried out by different people, while not necessarily in different areas. For this reason these functions are represented twice at the model. They are: <Seek for Emergency Care>; $<$ Admission, dressing, and snack rooms >; <Triage >; <Wait for Medical Consultation>; $<$ Medical Evaluation>; <Tomography Exams>; <Provide Emergency Care>; <Transport Patients>; <Carry out surgery>; <Provide Ward Care>; <Provide ICU Care>; and $<$ Patient Discharge $>$. The physical space where each function is carried out is represented in Figure 1 by the coloured rectangles on the background. In fact, two functions, namely<Transport Patients> and <Wait for Medical Consultation> occur in shared spaces 
such as certain corridors, elevators, and rooms in the emergency department. Some functions were new and specifically created to face the pandemic. These functions are: $<$ donning and doffing PPE>; <lab tests>; <provide COVID-19 care - emergency >; $<$ collect samples for exams (COVID-19)>; <provide COVID-19 care - ICU>; <Provide COVID-19 care ward>.

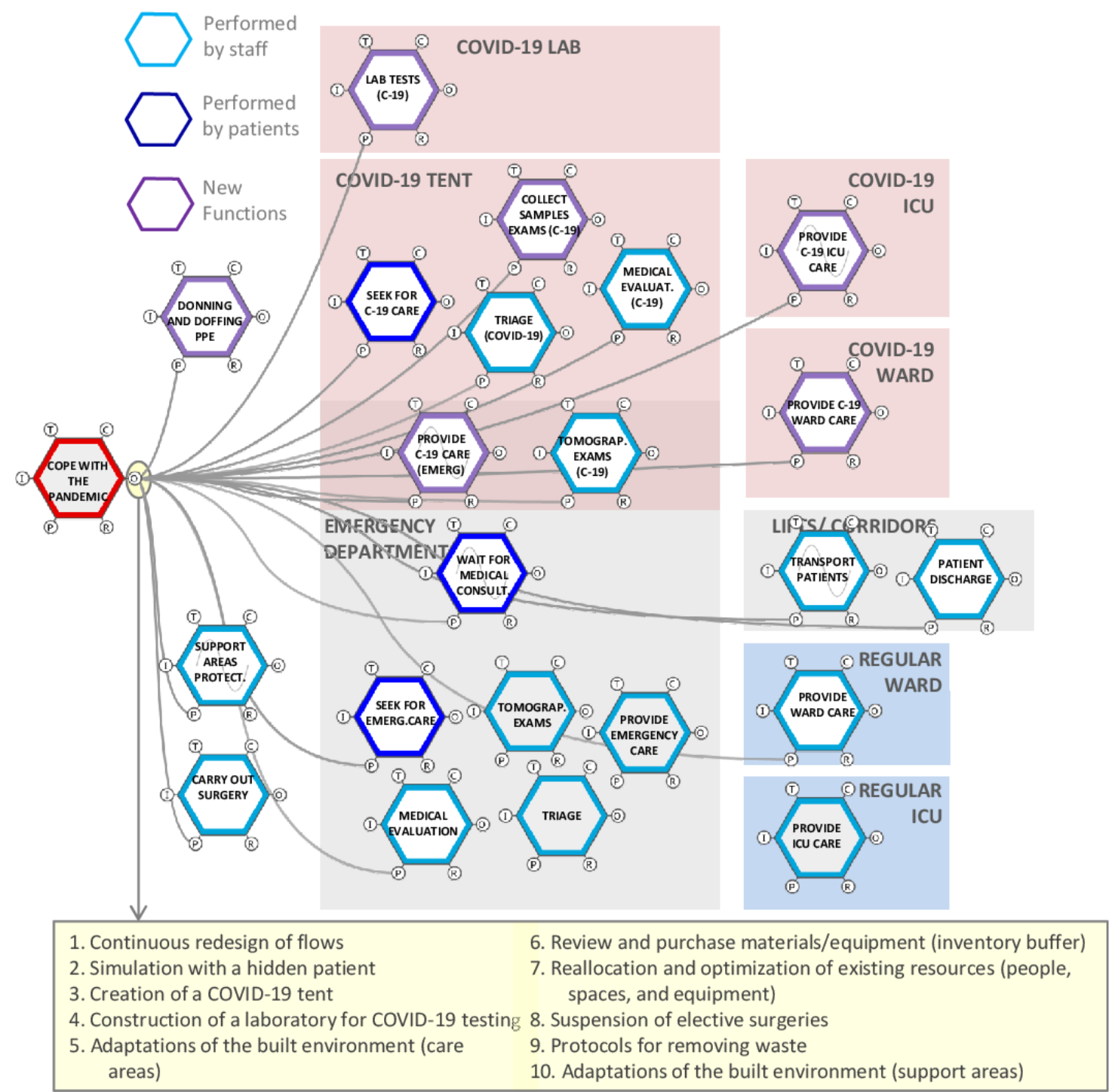

Figure 1 - FRAM model highlighting the outputs of the function <Cope with the pandemic $>$ performed by the COVID-19 Committee.

The sequencing of the functions varies according to the circumstances. For instance, depending on the patient's condition, they may need to undertake a tomography exam, a surgery, and then be transferred to the ICU. Others will be discharged after receiving treatment at the emergency department. Thus, the journey of both COVID and nonCOVID patients can involve, broadly speaking, a mix of emergency, ward, and ICU care. Furthermore, patients can change from COVID to non-COVID (and vice versa) after being hospitalized. 
The built environment's influence on resilience of healthcare services: lessons learnt from the COVID-19 pandemic

\section{Resilience Practices to Cope With the Pandemic}

The hospital adopted measures to cope with the pandemic even before the first infected patient was admitted in March 2020. Since then and up to the time of writing this article, a multidisciplinary committee for combating coronavirus, hereafter named COVID-19 committee has been in charge of assessing the threats imposed by the pandemic and developing plans for the provision of the necessary resources (e.g., staff, materials, and space). It is a multidisciplinary team composed mainly of hospital units' managers (e.g., emergency, ward, ICU, infection control service, risk management). This team meets on a daily basis. The committee and the hospital have a strong culture of involving professionals before making important decisions. Thus, whenever needed, they consult workers from care units (e.g., nurses and physicians) and administrative positions, such as infrastructure managers.

One of the regular activities of this committee has been the continuous redesign of flows (output 1) of patients and resources. Indeed, since March 2020 the staff was aware of the need for the design of dedicated clinical (e.g. triage) and non-clinical (e.g., waste disposal) pathways to COVID-19. An important event that took place at the hospital two months before the first case of a COVID-19 patient in Brazil was the simulation with a hidden patient (output 2) hypothetically infected with the new coronavirus. In fact, this activity was mandatory as the hospital is accredited by the Joint Commission International (JCI) and, for that reason, it is required to carry out an annual verification of preparedness for emerging global diseases. A managerial team was formed to draw the simulation flow. The hidden patient started their journey at the emergency department in which staff members were supposed to identify whether or not the patient was infected with COVID19. Thus, on each step passed by the patient, the managerial team gave feedback to frontline healthcare workers on how to properly respond. All interviewees agreed that this simulation allowed for the identification of vulnerabilities and opportunities to adjust hospital flows. Another action taken by the COVID-19 committee was the creation of a COVID-19 tent (output 3) outside of the main hospital building and nearby the entrance to the emergency department in order to triage suspected cases. Furthermore, the managerial team realized that the outsourced laboratory was not providing timely results of COVID-19 tests (function <Lab Tests> (output 4). Then, resources were set up for the construction of the hospital's own COVID-19 test laboratory in order to reduce the processing lead time of tests from 4 days to no more than 24 hours (Polanczyk et al., 2020).

A crucial resource available to respond to COVID-19 patient flows was the area previously occupied by the paediatric emergency department, which had recently moved to another building inside the same hospital site. This change occurred before the onset of the pandemic and luckily allowed for the just-on-time expansion of the existing adult emergency department (output 1). As part of these changes, an exam room dedicated to the testing of COVID-19 suspected patients was set up within the emergency department (output 5). However, some of the spaces could not be totally separated between COVID and non-COVID patients, which was the case of the room where the function <Wait for Medical Consultation> occurred. This situation posed variability as non-infected patients could be infected while waiting for the consultation; there was only a curtain separating beds.

Although the separation of COVID and non-COVID patient flows was imagined in design, it was challenging to be maintained that way all the time. A core function that addressed breaches in that design was <Donning and Doffing PPE>, which should be 
carried out by all employees immediately after entering and leaving the hospital building. Safeguards for coping with the impossibility of fully separate flows were also adopted for the function <Transport patient> when it occurred in the elevator. The initial plan was to designate a dedicated elevator for COVID-19 patients, but as they would move across public areas of the hospital and as flows were constantly changing, the decision was made to allow the circulation in the same elevators, although not at the same time. Elevators were frequently cleaned and people inside were wearing personal protective equipment (PPE).

Regarding the function <Provide COVID-19 ICU Care> (output 5), the committee firstly decided to transfer the Bone Marrow Transplant (BMT) Unit to another location in order to use that space to create 22 ICU beds to COVID-19 patients. This area was selected due to its physical attributes, such as the high quality of the Heating, Ventilation, and Air Conditioning (HVAC) system. However, the airflow required for the treatment of patients subject to BMT was the opposite to that required by COVID patients - i.e., the former needs to block airflow from the outside air, while the latter needs to block airflow to the outside air - this latter is referred to as negative air pressure areas. In common, both situations demand equipment to provide air renovation to match their specific needs. Thus, it was easier for the infrastructure team to change the air direction in that area than in other units with no existing similar air renovation facilities. In addition, High-Efficiency Particulate Air (HEPA) filters were installed as a barrier to the coronavirus spread in those units. Initially, the idea was to place COVID-19 patients in isolated rooms with negative air pressure, divided by medical specialty. However, this would imply in maintaining COVID-19 patients dispersed in several units, which would also increase infection opportunities during inter-unit flows. As a result, the committee created a few hubs of infected patients, all of them with the proper HVAC system's adaptation.

The BMT unit occupation was particularly useful during the early stages of the pandemic as it provided a window of time for the committee to plan other changes in the hospital flows and physical areas. Even though that unit played a key role, the lack of visibility among rooms had a negative impact on staff performance. The area was not originally designed to support ICU functions and interviewees reported that the rooms did not have the necessary visibility to allow staff to communicate and work collaboratively. This hindered the abilities of monitoring the processes and anticipating events. Thus, as time passed, that unit was found not appropriate for COVID patients, which were then transferred to other areas.

Since the hospital's physical structure cannot further expand, the management of buffers of materials and equipment as well as the reallocation and optimization of existing resources (people, spaces, and equipment) (outputs 6 and 7) have been vital. For instance, the committee decided to suspend the elective surgeries early in the outbreak to increase the availability of beds for COVID-19 patients, readmitting the most acute patients gradually (output 8). Additionally, individual rooms were transformed into shared rooms. Extra equipment and materials have been acquired to the possible extent, especially PPE, medications, and mechanical ventilators. These measures have been stopped and reinstated cyclically as the pandemic evolves in order to free up resources during the most critical periods.

Regarding the protocols for removing waste (output 9), they addressed the activities of the cleaning staff, more specifically when they picked up the bags of dirty clothing, threw them into trolleys and then moved them to the laundry. These activities pose a risk 
The built environment's influence on resilience of healthcare services: lessons learnt from the COVID-19 pandemic

of environmental contamination, as throwing the bags can produce aerosol. The committee established measures to monitor these activities and the cleaning staff is now required to perform a different procedure. They collect the dirty clothes from COVID-19 units at the end of their shift, wearing a specific apron and label the plastic bags to make it clear that it stems from a COVID-19 unit. Some built environment adaptations in the administrative and support areas (output 10) were a precondition to the function $<$ Support areas protection>. For example, glasses were installed in all hospital reception desks to protect workers and patients. Other measures involved the separation of the dressing room for COVID and regular staff and changes in the layout of the staff room to ensure social distancing and prevent gatherings.

\section{DISCUSSION}

Six main lessons can be learnt from the outputs of the FRAM function <Cope with the pandemic $>$ (Figure 1). The lessons are described according to the design and operation phase of the building. Thus, lessons to the design phase are primarily targeted at the BEAI, while lessons to the operation phase are applicable to the BEAD. The lessons are logically related to the four resilience abilities.

\section{Lessons to the Design Phase (BEAI)}

The lessons learnt for the building design phase are mostly related to the resilience abilities of anticipating and responding. Indeed, the life cycle of buildings extends for decades or centuries. Although anticipation is challenging at the long-term, major threats such as pandemics are expected and therefore the building design must support prepared responses. The main lessons learnt are presented below and they resulted from both insights from the literature and difficulties experienced by the studied hospital - i.e., the proposals embedded in the lessons learnt were not fully accounted for in the design phase of the hospital building.

- To design flexible workspaces that can accommodate functions other than the primary functions (Capolongo et al., 2020; Saurin, 2021). For instance, wards are used primarily for the hospitalization of regular in-patients. However, the case study indicated that designers could anticipate the need for attending patients with breathing difficulties that need extra oxygen supply in the ward (output 5). In this case, the built environment should allow for the quick expansion of the HVAC systems, the adjustment of air direction, and the easy flow of the medical gases throughout the building structure (Gordon et al., 2020; Capolongo et al., 2020). These infrastructures need walls on which they can be inserted or attached, which are not easily available in all areas. In other hospitals in Brazil and elsewhere, the lack of walls nearby the beds has implied the need for using oxygen tanks; and

- To design the main hospital entrance, emergency department, and a portion of the intensive care units, preferably on the same floor, in order to shorten the flow of infected patients and therefore reducing the possibility of contagion (Capolongo et al., 2020). For buildings with multiple floors, an alternative solution might be the design of dedicated elevators for patients with highly contagious diseases, which was a measure considered but not implemented in the case study. 


\section{Lessons to the Operation Phase (BEAD)}

The lessons for the operation phase of the building are mostly related to the resilience abilities of monitoring and responding. Monitoring might point out gaps between the BEAI and the BEAD, triggering responses to threats unanticipated in the design phase. Four main lessons can be mentioned in this regard. Differently from the lessons related to the design phase, those for the operation phase reflect strategies that were in place at the studied hospital.

- To save financial resources for acquiring scarce supplies in a competitive market as well as to maintain a multi-skilled workforce to cope with demand surges. Purchasing drugs, equipment, construction of new spaces (e.g., laboratory and COVID-19 tent), refurbishment or adaptation of existing facilities (outputs 4, 5, and 6) are costly measures that might be necessary to cope with unexpected events (Achour and Price, 2010; Polanczyk et al., 2020; Capolongo et al., 2020);

- To develop internal capabilities for the best use of available resources, which includes their quick reallocation when necessary (output 7) as well as combining short-term and long-term thinking. In the studied hospital those capabilities were mainly represented by the committee formed in the early stages of the pandemic. An example of reallocation of resources refers to the suspension of elective surgeries (output 8), allowing for the reallocation of beds and staff to other hospital units. Similarly, the telemedicine resources that have been used by family members to virtually interact with ICU patients will be used after the pandemic for the same purpose;

- To use visual management strategies to quickly and publicly announce changes in the built environment, avoiding misunderstandings that may put workers and patients at risk of contamination. This practice, which reinforces the importance of redundant information, was suggested by one of the nurses interviewed who had witnessed a co-worker inadvertently entering a COVID area without being aware of that. To respond to this situation, investments in wayfinding in hospital flows are necessary when changing routes and units functioning (Capolongo et al., 2020); and

- To strengthen a collaborative organizational culture by encouraging multidisciplinary committees in charge of monitoring processes and deploying quick responses to unexpected events (e.g., COVID-19 Committee).

\section{CONCLUSIONS}

This paper offers an exploratory report of how the COVID-19 pandemic has demanded resilience from healthcare services, emphasizing built environment implications. Lessons learnt were identified for the design and operation phase of healthcare facilities. These lessons are likely to be of interest not only to the studied hospital but also for others facing similar challenges around the world. The lessons were related to the resilience abilities of anticipating, monitoring, and responding. In fact, it is worth noting that these lessons were compiled by the researchers and therefore we are not certain of the extent to which they have been actually learnt by the studied healthcare organization and will be used in the development of new procedures of care and building designs.

All of the lessons learnt were underpinned by the problem of matching capacity to demand. Therefore, the design of flexible workspaces (e.g., possibility of installing ICU 
The built environment's influence on resilience of healthcare services: lessons learnt from the COVID-19 pandemic

equipment in regular patient wards) stands out as a major theme. Further work will focus on updating the lessons learnt as the pandemic evolves and retrospective in-depth studies after it subsides.

\section{REFERENCES}

Achour, N., Price, A.D.F., (2010). "Resilience strategies of healthcare facilities: present and future."Int. J. Disaster Resilience Built. Environ. 1 (3), 264-276.

Bosher, L., Carrillo, P., Dainty, A., Glass, J., Price, A., (2007). "Realising a resilient and sustainable built environment: towards a strategic agenda for the United Kingdom.”Disasters 31 (3), 236-255.

Capolongo, S.; Gola, M.; Brambilla, A.;Morganti, A.; Mosca, E. I.; Barach, P. (2020). "COVID-19 and Healthcare Facilities: a Decalogue of Design Strategies for Resilient Hospitals". ActaBiomed., Vol. 91, Supplement 9: 50-60. doi.org/10.23750/abm.v91i9-s.10117.

Clay-Williams. R.; Hounsgaard, J.; Hollnagel, E. (2015). "Where the rubber meets the road: using FRAM to align work-as-imagined with work-as-done when implementing clinical guidelines."Implementation Science, 10(1), 125.

Cilliers, P. I. (1998). Complexity and postmodernism: understanding complex systems., London: Routledge.

Gordon, D.; Ward, J.; Yao, C. J.;Lee, J. (2020). "Built Environment Airborne Infection Control Strategies in Pandemic Alternative Care Sites." HERD: Health Environments Research \& Design Journal, 1937586720979832.

Hollnagel, E. (2012). FRAM: The Functional Ressonance Analysis Method - modelling complex socio-technical systems. Burlington: Ashgate.

Hollnagel, E., Braithwaite, J., Wears, R., (2013). Resilient Health Care. Ashgate, Burlington.

Hollnagel, E. (2017). Safety-II in practice: developing the resilience potentials. Routledge.

Keenan, J. M. (2020). "COVID, resilience, and the built environment."Environment Systems and Decisions, v. 40, p. 216-221.

Polanczyk, Carisi A. et al.(2020). "Getting Ready for the Covid-19 Pandemic: Experience of a Brazilian Hospital."NEJM Catalyst Innovations in Care Delivery, v. 1, n. 5.

Ransolin, N.; Saurin, T. A.; Formoso, C. T. (2020)a. "Integrated modelling of built environment and functional requirements: Implications for resilience." Applied Ergonomics, 88, 103154.

Ransolin, N., Saurin, T.A.;Formoso, C.T. (2020)b."Influence of the Built Environment on Patient Safety and Well-being: a Functional Perspective." In: Tommelein, I.D. and Daniel, E. (eds.). Proc. 28th Annual Conference of the International Group for Lean Construction (IGLC28), Berkeley, California, USA.

Yin, R. (2014). Case Study Research: Design and Methods (5 th ed.). Los Angeles, CA: SAGE.

White, M. D., \& Marsh, E. E. (2006). Content analysis: A flexible methodology. Library trends, 55(1), 22-45. 